\title{
Shape-Similarity Search of Three-Dimensional Models Using Parameterized Statistics
}

\author{
${ }^{1}$ Ryutarou Ohbuchi, ${ }^{1}$ Tomo Otagiri, ${ }^{2}$ Masatoshi Ibato, ${ }^{1}$ Tsuyoshi Takei \\ ohbuchi@acm.org, k7022@kki.yamanashi.ac.jp, zap5490@cat.zero.ad.jp, f8058@kki.yamanashi.ac.jp \\ ${ }^{1}$ Computer Science Department, Yamanashi University, 4-3-11 Takeda, Yamanashi-shi, Japan \\ ${ }^{2}$ Ibaraki Computing Center, Incorporated.
}

\begin{abstract}
In this paper, we propose a method for shape-similarity search of $3 D$ polygonal-mesh models. The system accepts triangular meshes, but tolerates degenerated polygons, disconnected component, and other anomalies. As the feature vector, the method uses a combination of three vectors, (1) the moment of inertia, (2) the average distance of surface from the axis, and (3) the variance of distance of the surface from the axis. Values in each vector are discretely parameterized along each of the three principal axes of inertia of the model. We employed the Euclidean distance and the elastic-matching distance as the measures of distance between pairs of feature vectors. Experiments showed that the proposed shape features and distance measures perform fairly well in retrieving models having similar shape from a database of VRML models.
\end{abstract}

Keywords: content-based search and retrieval, geometric modeling, polygonal mesh, principal axes, elastic matching.

\section{Introduction}

With the recent increase in the number of threedimensional (3D) geometric models, both on the Internet and in the local storage media at movie studios and automobile manufacturers, development of the technology for effective content-based search and retrieval of threedimensional (3D) models has become an important issue. Content-based search and retrieval of text, audio and image data has been actively studied for many years. In case of 3D geometric models, however, investigation into contentbased search has gained attention only recently.

A 3D model could be searched by textual annotation by using a conventional text-based search engine. This approach wouldn't work in many of the application scenarios. The annotations added by human beings depend on culture, language, age, sex, and other factors. It is thus necessary to have a content-based search and retrieval systems for $3 \mathrm{D}$ models that are based on the features intrinsic to the 3D models, most important of which is the shape [Paquet97, Suzuki98, Keim99, Regli00, Suzuki00, McWherter01, Osada01, Novotni01, Hilaga01, Vranic01, Corney02, Mukai02].

A typical method for shape similarity search of 3D geometric models consists of three steps, (1) the computation of a set of shape features from a given model, (2) the computation of distance among pairs of shape features, and (3) the retrieval of the model based on the distance values, e.g., $k$-nearest neighbors.

One of the issues that need consideration is the shape representation(s) that the system intends to accept. There are wide variety of 3D shape representations, such as solids bounded by parametric curved surfaces or polygons, voxel enumeration, sum of implicit functions, or VRML-like "polygon-soup", to name a few. There is no single 3D shape representation or format that could serve as a greatest common denominator to all 3D shape representations. Only a small subset of the shape representation pairs is compatible enough so that they can be converted back and forth with reasonable approximation. For example, a solid model can be converted into a voxel representation with reasonable approximation. However, a VRML model, which in general does not define a solid, can be converted to neither voxel nor "watertight" meshes. Two-dimensional (2D) images, on the other hand, has 2D array of pixels as the greatest common denominator of $2 \mathrm{D}$ image representations.

Many 3D shape similarity comparison algorithms, for example, those by Hilaga [Hilaga01] and Vranic [Vranic01], assume as its input a solid bounded by a polygonal mesh. A majority of recent mechanical CAD models define solids bounded by curved surfaces, and are very well defined. Regli [Regli00], McWherter [McWherter01], Corney [Corney02], and Mukai [Mukai02] are the examples of shape similarity search methods directed toward geometrical CAD models. Keim et al. [Keim99] dealt with the voxel enumeration 
representation. These are relatively well-defined shape representations for they represent solid.

We aim at a shape similarity search system that can handle VRML-like models. To search for models represented as VRML models, one must deal with an illdefined shape definition often referred to as "polygon soup". A polygon soup defines model that gives a visual impression of 3D shapes by using a collection of polygonal meshes, independent polygons, line fragments, and points. It does not define "proper" 3D objects, i.e., solids. While triangular meshes are the primary target, the methods by Osada et al. [Osada01] and Elad et al. [Elad01] allow degeneracies in the meshes, such as zero-area triangle and disconnected components. The algorithm described in this paper also targets the same class of shape representation as the methods by [Osada01] or Elad et al. [Elad01].

Given a model defined using a shape representation, a typical shape similarity search system extracts a succinct shape description or feature for shape similarity (or dissimilarity) comparison. Most of the shape features employed so far are of geometrical nature [Paquet97, Suzuki98, Keim99, Suzuki00, Osada01, Novotni01, Vranic01]. One of the first studies on shape similarity search published by Eric Paquet et al. [Paquet97] employed two kinds of geometrical features and various photometric properties for similarity measurement. Photometric properties included color, reflectance, and texture. The first geometric feature is the histograms of angles between the surface normal vectors and the first two of the principal axes. The second geometric feature is a set of statistics computed from "cords". A cord is a vector from the center of mass of the model to the center of mass of a triangle. If the shape is solid, i.e., if an inside-outside test can be performed, the model can be converted to a voxel-based representation for feature extraction and distance computation [Novotni01]. Or, the shape may be given in a voxel representation to start with [Keim99]. Hilaga et al. [Hilaga01] employed topology as the primary shape feature in its shape similarity query. Their topological feature is based on the Reeb graph. However, unlike the traditional Reeb graph, Hilaga's topological feature can be computed independent of the location and orientation of the shape. Using Hilaga's method, shapes having different topology, for example, a torus and a sphere, can be distinguished clearly. It could also recognize a pair of geometrically different but topologically similar shapes, e.g., human figures having either bent or straight elbows. Note that Hilaga's method can't compare a 3D model that consists of more than one (topologically) disconnected components, e.g., two detached spheres, for his topological analysis can't be performed on such a model.

Given a pair of shape features, similarity, or more commonly, dissimilarity or distance between the two features are computed so that the distance among pairs of objects can be ranked. The best way to compute distance is yet to be found, given a wide variety of shape features and the difficult nature of the comparison. Relatively simple distance measures include Euclidean distance, Manhattan distance, and Hausdorff distance. In the field of contentbased image search and retrieval, many more distance measures have been studied [Veltkamp01]. Another possible approach is to employ a trained classifier, e.g., a neural network or a Support-Vector Machine (SVM) [Elad01] to find the models close to the queried shape.

The method to pose a query by itself is a difficult problem for a 3D shape similarity search. The most obvious way is to present an example 3D model (or a set of example models) and tell the system to find shapes similar to it. This approach is probably sufficient for many applications. Another approach could be to manually draw an example shape to be searched. It is relatively easy to draw a 2D figure, or a set of 2D figures (e.g., orthographic projections) of the $3 \mathrm{D}$ shape sought for. But a projection or projections of $3 \mathrm{D}$ shape onto $2 \mathrm{D}$ contour would introduce ambiguity into the query. Furthermore, drawing is not an easy task for many people. It is also possible to draw 3D shapes directly, for example, by using a 3D shape sketch tool similar to the Teddy [Igarashi99]. However, drawing in $3 \mathrm{D}$ is tougher than drawing in 2D for most of the people. And drawing topologically complex $3 \mathrm{D}$ shapes, such as those having holes and branches can be quite difficult. For the system described in this paper, we have settled for the easiest method, that is, querying by using a 3D model given a priori as the example.

As the distance computed and the models retrieved, one finds another issue to be solved, the issue of subjectivity in shape similarity comparison. Experiments using our proofof-concept system showed that the models the system chose as the closest to the query presented are not necessarily the models the user wanted. This discrepancy in part stems from the subjective nature of shape similarity decision. To accommodate such subjectivity, Suzuki et al. employed, in their pioneering work on 3D shape similarity search, multidimensional scaling [Suzuki98, Suzuki00] so that subjective keywords used in the query and the shape features computed from the 3D shapes are strongly correlated. Another approach is to employ a learning classifier such as the SVM for a human directed search [Elad00]. The SVM is a binary classifier that creates and uses a nonlinear hyper-plane with maximum margins to the training samples of the given pair of classes [Burges98, Vapnik98, Vapnik99]. Elad et al. computed, after pose normalization, statistical moments of points distributed on mesh surfaces for their feature vector. They fed the feature vector to an SVM to compute the dissimilarity. We also experimented with the SVM [Ibato02] by combining the pose-normalization free shape feature $\mathrm{D} 2$ defined by Osada 
et al. [Osada01] with the SVM.

In this paper, we propose and evaluate a set of shape features and a pair of shape dissimilarity measures for a shape similarity search system for 3D shapes defined as polygonal meshes. The shape features we propose are three statistics discretely parameterized along the principal axes of inertia of the model. To compute the inertial axes as well as the statistics, we assumed the triangular faces to have uniformly distributed mass, and approximated it by using a Monte-Carlo approach. As the dissimilarity measure between a pair of feature vectors, we experimented with the simple Euclidean distance and the elastic matching distance that took advantage of the dynamic programming for efficient computation. Experiments showed that our proposed methods have promising characteristics. While the proof-of-concept system is not yet ready for real-world applications, the components we have developed could be useful in a future shape-similarity search and retrieval system.

\section{Shape similarity search algorithm}

Our shape similarity search system assumes, as its inputs, 3D polygonal meshes. An entry in the database stores a 3D model along with a pre-calculated feature vector for the model. Currently, the database itself is organized as a simple array and has no indexing and other methods to accelerate search and/or retrieval.

As the query interface, we adopted the query-byexample approach. A user presents the system with an example 3D shape and asks the system to retrieve its $k$ nearest shapes.

The system first normalizes the location and orientation of the model using the center of mass and the principal axes of inertia of the model. The computations of the center of mass and the inertial moment are performed by assuming that the each polygonal surface has a uniform density, and approximating it by using a Monte-Carlo approach.

As the shape feature, our method employs three statistics that are discretely parameterized along the principal axes of inertia the model. To compute statistics parameterized along an inertial axis, the model is subdivided into slabs along the axis. Then, for each slab, compute; (1) the moment of inertia about the axis, (2) the average distance to the surfaces from the axis, and (3) the variance of distance to the surfaces from the axis.

A dissimilarity value among a pair of feature vectors is computed using two methods, the Euclidean distance and the elastic-matching distance. The latter is intended to accommodate certain local deformation of model's shape, e.g., elongation of the torso part of a horse model.

Details of each step of our shape similarity search method will be explained below.

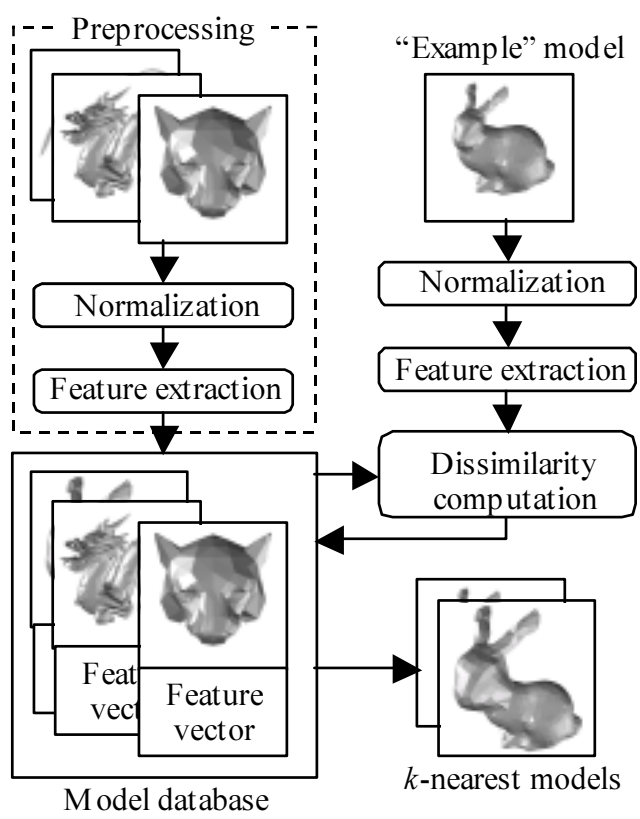

Figure 1. The block diagram of our shape-similarity search algorithm.

\subsection{Pose normalization}

Our method first normalizes the size and orientation of the model prior to extracting shape features. To normalize orientation, we compute principal axes of inertia. Gottschalk et al. assumed unit point mass located at each vertex and computed the principal axes of inertia [Gottschalk94]. The principal axes computed using this method depends of the tessellation of the mesh. In our approach, mass is assumed to be uniformly distributed on the surface of the mesh. We approximated it by placing a number of point masses at a random location on each triangle. The number of points is proportional to the area of the triangle. Using the point mass on the surface, the covariance matrix $\mathbf{C}$ can be computed using the formula;

$$
\mathbf{C}=\left(c_{p q}\right)=\frac{1}{n} \sum_{i=1}^{n}\left(p_{i}-p_{M}\right)\left(q_{i}-q_{M}\right)
$$

where $p, q \in\{x, y, z\}$. Three eigenvectors of the covariance matrix $\mathbf{C}$ are the principal axes of inertia of the model. We name the axes $\alpha, \beta$, and $\gamma$ in the decreasing order of the magnitude of the eigenvalues associated with each axis. We resolve the issue of symmetry of the principal axes by using the distribution of points (i.e., mass) about the center of mass the model.

To generate points on triangles, we adopted the method by Osada et al. [Osada01]. Using Osada's method, given the coordinates of the vertices of the triangle $\mathbf{t}_{1}, \mathbf{t}_{2}$, and $\mathbf{t}_{3}$ and a pair of pseudo-random number sequences (PRNS) $r_{1}$ and $r_{2}$, the coordinate of the point $\mathbf{P}$ is computed as 
follows.

$$
\mathbf{P}=\left(1-\sqrt{r_{1}}\right) \mathbf{t}_{1}+\sqrt{r_{1}}\left(1-r_{2}\right) \mathbf{t}_{2}+\sqrt{r_{1}} \quad\left(r_{2} \cdot \mathbf{t}_{3}\right) .
$$

Actually, instead of the PRNS $r_{1}$ and $r_{2}$, we used a lowdiscrepancy sequence or quasi-random number sequence (QRNS) Sobol [William92] for a more stable and lowererror alignment of the model's orientation. Figure 2 shows the results of an experiments that compared the angular alignment error (in degrees) using the PRNS (the drand48() library function) and the Sobol QRNS with the formulae (1) and (2). The error figure is the average of three angular errors, in degree, of the three axes $\alpha, \beta$, and $\gamma$ over 10 experimental runs using the bunny model of Figure 4. It can be seen that the Sobol QRNS consistently outperformed the drand48() PRNS.

The point masses generated on the triangles are also used to compute statistics to be used as shape features, which will be explained below.

\subsection{Parameterized statistics}

Our shape features are three vectors of statistical values that are discretely parameterized along one of the principal axes of inertia. The statistics are, (1) the moment of inertia about the axis, (2) the average distance to surfaces from the axis, and (3) the variance of distance to surfaces from the axis. The algorithm computes 9 vectors in total; three statistics along each of the three the principal axes. The dissimilarity (or distance) computation uses a vector that is a concatenation of these 9 vectors.

To compute a feature vector discretely parameterized along an axis, the model is segmented into $l$ equal thickness slabs along the axis by $(l-1)$ parallel, equal interval planes perpendicular to the axis (See Figure 3). The $i$ th component of the (l-1)-dimensional feature vector is computed from the ith analysis window, which is a pair of adjacent intervals at the positions $i$ and $(i+1)$. If we have $l$ slabs, there are (l-1) analysis windows and the feature vector is (l-1)-dimensional. This overlap of analysis windows makes the feature vector somewhat more robust against slight alignment errors or minor geometric distortions along the axis.

A Monte-Carlo approach is used again in computing the statistics; the points generated on the surfaces of the mesh for the pose normalization (Section 2.1) are used again for the computation of the statistics.

Let $\mathbf{m}_{\alpha, i}, \mathbf{m}_{\beta, i}, \mathbf{m}_{\gamma, i}$ be the inertial moment about the axes $\alpha, \beta$, and $\gamma$, respectively, of the $i$-th $(1 \leq i \leq l-1)$ analysis window (i.e., a pair of adacent intervals at positions $i$ and $(i+1)$ ). Similarly, let $\mathbf{a}_{\alpha, i}, \mathbf{a}_{\beta, i}, \mathbf{a}_{\gamma, i}$ and $\mathbf{d}_{\alpha, i}, \mathbf{d}_{\beta, i}, \mathbf{d}_{\gamma, i}$, be the average and variance of the distance of surfaces from the axis $\alpha, \beta$, and $\gamma$, respectively, of the $i$ th $(1 \leq i \leq l-1)$ analysis window. The feature vector $\mathbf{F}$ for

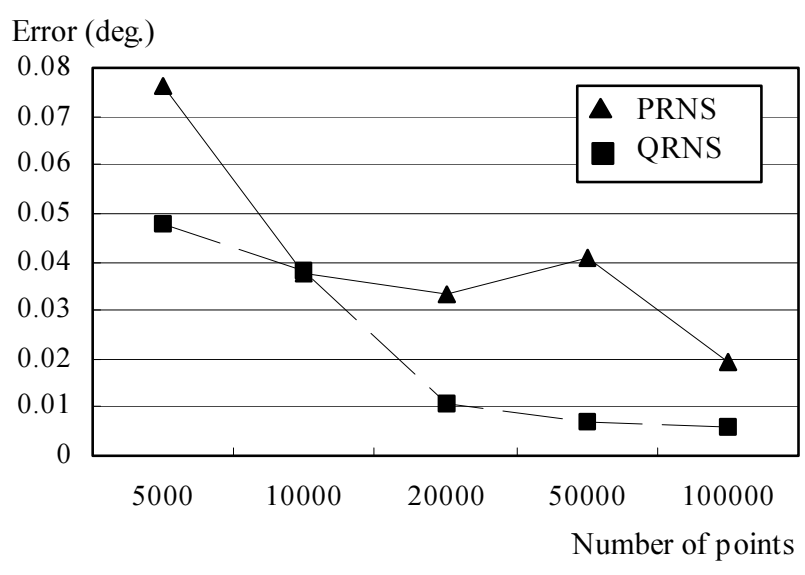

Figure 2. The average angular error after pose normalization computed using the pseudo-random number sequence (PRNS) and quasi-random number sequence (QRNS).

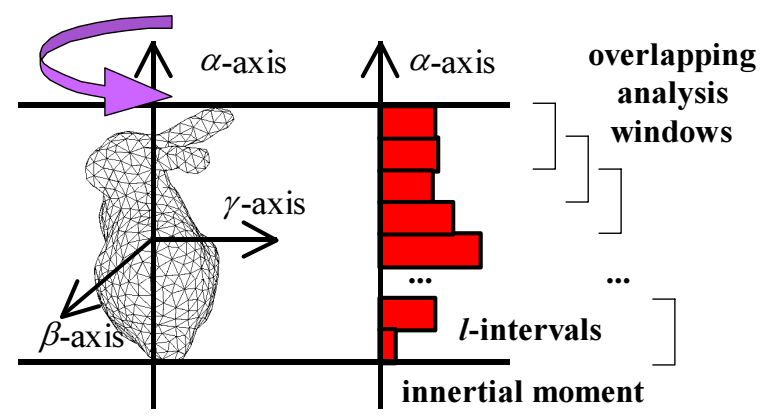

Figure 3. Computation of the moment parameterized along the (most-) principal axis $\alpha$.

the model M becomes;

$$
\mathbf{F}=\left(\mathbf{F}_{p}\right)=\left(\begin{array}{c}
\mathbf{m}_{p} \\
\mathbf{a}_{p} \\
\mathbf{d}_{p}
\end{array}\right)=\left(\begin{array}{c}
m_{p, 1}, m_{p, 2}, \cdots, m_{p, l-1} \\
a_{p, 1}, a_{p, 2}, \cdots, a_{p, l-1} \\
d_{p, 1}, d_{p, 2}, \cdots, d_{p, l-1}
\end{array}\right)
$$

where $p \in\{\alpha, \beta, \gamma\}$ indicates the axis.

For the slab $i$ along the axis $p$, the three components of the vector $\mathbf{F}$, that are, the moment $m_{p, i}$, the average distance of points from the axis $a_{p, i}$, and the variance of distances of points from the axis $d_{p, i}$ are computed by the following formulae. In the formulae, $d_{p, i}$ is the distance to the point $j$ on the surface from the axis $p$, and $n$ is the number of points in the slab $i$.

$$
\begin{aligned}
& m_{p, i}=\sum_{j=1}^{n} m r_{j}^{2} \\
& a_{p, i}=\frac{1}{n} \sum_{j=1}^{n} r_{j}
\end{aligned}
$$




$$
d_{p, i}=\frac{1}{n-1} \sum_{j=1}^{n}\left(r_{j}-a_{p, i}\right)^{2}
$$

We then normalize the magnitude of the feature vector $\mathbf{F}$ so that $\left\|\mathbf{m}_{p}\right\|=\left\|\mathbf{a}_{p}\right\|=\left\|\mathbf{d}_{p}\right\|=1$. This process is repeated for each of the three axes $\alpha, \beta$, and $\gamma$.

Figure 4 and Figure 5 show an example of a set of feature vectors parameterized along the principal axis of inertia $\alpha$ using the number of analysis windows $l=15$.

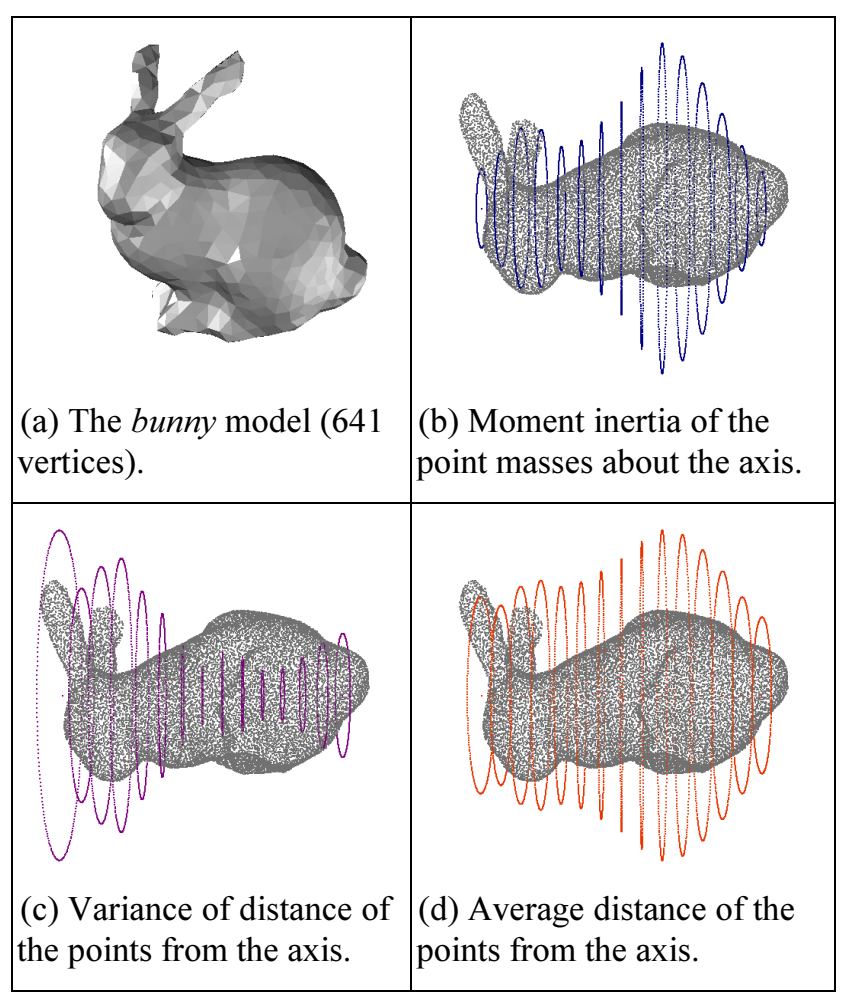

Figure 4. Examples of the statistics parameterized along the axis $\alpha$. The number of analysis windows $(l-1)=15$.

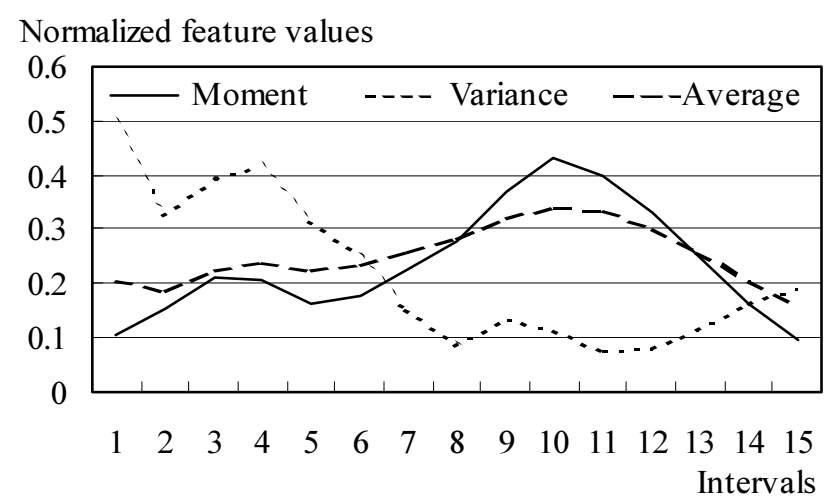

Figure 5. Plots of the three parameterized statistics for the bunny model used in Figure 4.

\subsection{Dissimilarity computation}

Similarity, or, more appropriately, dissimilarity among a pair of model is computed as the distance between their feature vectors. We employed two distance measures, the Euclidean distance and the elastic matching computed efficiently by using dynamic programming.

The dissimilarity $D(\mathbf{X}, \mathbf{Y})$ is computed by summing the distance values for all of the principal axes of inertia.

$$
D(\mathbf{X}, \mathbf{Y})=\left(\sum_{p \in\{\alpha, \beta, \gamma\}}\left(d\left(\mathbf{X}_{p}, \mathbf{Y}_{p}\right)\right)^{2}\right)^{\frac{1}{2}}
$$

Note that the statistics may be weighted when computing the dissimilarity. Weighting can be done by the statistics, or by the principal axis. For example, dissimilarity values may be computed without using the standard deviation of the distance $\mathbf{d}_{\alpha}, \mathbf{d}_{\beta}, \mathbf{d}_{\gamma}$, using $\mathbf{m}_{\alpha}, \mathbf{m}_{\beta}, \mathbf{m}_{\gamma}$ and $\mathbf{a}_{\alpha}, \mathbf{a}_{\beta}, \mathbf{a}_{\gamma}$ only. Doing so will retrieve models whose overall shape are similar to the queried shape, paying less attention to the minor protrusions or dips such as noise or antennas of an ant that would increase the standard deviation.

Euclidean distance. Orientations of the models affect the distance value computed among a pair of models. If the principal axes of a two identical (or similar) models are parallel but one of the axes is pointing $180 \mathrm{deg}$. away from the other (Figure 6), the computed distance value will be much larger than when both axes were pointing the same direction. (Note that Figure 6 is for illustration purposes only. This problem occur only for models that are much more symmetrical.) To avoid this problem, we compute two distance values for both of the cases and take the

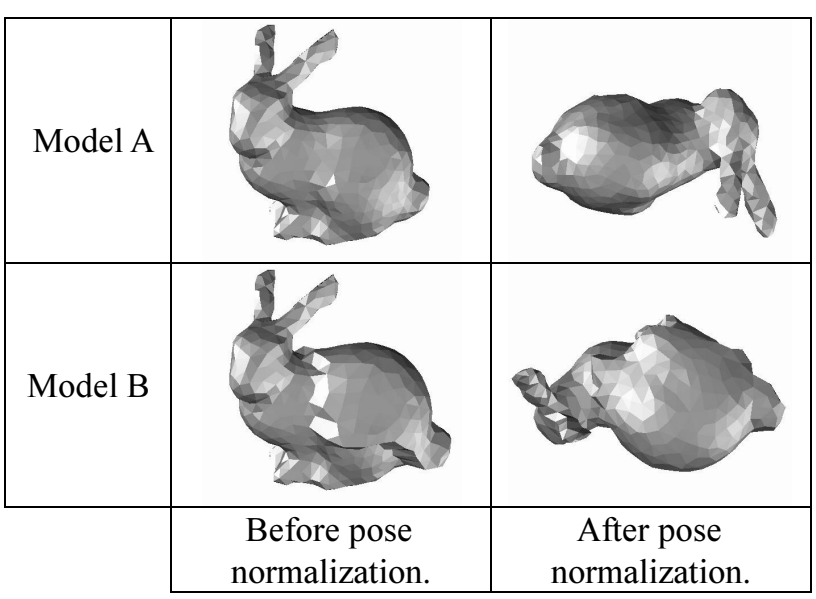

Figure 6. An example of principal axes that are parallel but pointing $180 \mathrm{deg}$. away from each other after pose normalization. 
minimum of the two.

The Euclidean distance $d_{\text {euc }}\left(\mathbf{X}_{p}, \mathbf{Y}_{p}\right)$ between two feature vectors $\mathbf{X}$ and $\mathbf{Y}$ are defined as below.

$$
d_{\text {euc }}\left(\mathbf{X}_{p}, \mathbf{Y}_{p}\right)=\min \left[\begin{array}{c}
\frac{1}{l-1} \sum_{i=1}^{l-1}\left(\left|\mathbf{X}_{p, i}-\mathbf{Y}_{p, i}\right|^{2}\right)^{\frac{1}{2}} \\
\frac{1}{l-1} \sum_{i=1}^{l-1}\left(\left|\mathbf{X}_{p, i}-\mathbf{Y}_{p, l-i}\right|^{2}\right)^{\frac{1}{2}}
\end{array}\right],
$$

where $p \in\{\alpha, \beta, \gamma\}$ are the axes of parameterization and $l$ is the number of intervals.

Elastic-matching distance. Euclidean distance is very "rigid" and could result in a larger-then-wanted distance value in some cases. For example, if the torso of an animal model is somewhat longer than the other animal model with identical head and tail parts, simple Euclidean distance measure will give a large distance value. Figure 7 shows a pair of models that are similar by a part of one of the model has been enlarged. (The bunny-bighead model was created by enlarging the head part of the bunny model.)

To partially compensate for this effect, we employed the elastic-matching distance computed by using dynamic programming. In the formulae below, $p \in\{\alpha, \beta, \gamma\}$ are the axes of parameterization, $l$ is the number of intervals, and $k=l-1$ is the number of analysis windows.

$$
\begin{gathered}
d\left(\mathbf{X}_{p}, \mathbf{Y}_{p}\right)=\frac{1}{2 k} g\left(\mathbf{X}_{p, l-1}, \mathbf{Y}_{p, l-1}\right) \\
g\left(\mathbf{X}_{p, k}, \mathbf{Y}_{p, k}\right)=\min \left[\begin{array}{l}
g\left(\mathbf{X}_{p, k}, \mathbf{Y}_{p, k-1}\right)+\Delta g\left(\mathbf{X}_{p, k}, \mathbf{Y}_{p, k}\right) \\
g\left(\mathbf{X}_{p, k-1}, \mathbf{Y}_{p, k-1}\right)+2 \Delta g\left(\mathbf{X}_{p, k}, \mathbf{Y}_{p, k}\right) \\
g\left(\mathbf{X}_{p, k-1}, \mathbf{Y}_{p, k}\right)+\Delta g\left(\mathbf{X}_{p, k}, \mathbf{Y}_{p, k}\right)
\end{array}\right] \\
\Delta g\left(\mathbf{X}_{p, i}, \mathbf{Y}_{p, j}\right)=\left(\left|\mathbf{X}_{p, i}-\mathbf{Y}_{p, j}\right|^{2}\right)^{\frac{1}{2}}
\end{gathered}
$$

In the field of speech recognition, prior to the advent of Hidden Markov Model-based methods, the elasticmatching distance was the standard in comparing a pair of phoneme sequences.

As is with the Euclidean distance, the distance values depend on the two possible orientation of the model. Note also that the elastic-matching distance is asymmetric; distance from model $\mathrm{X}$ to $\mathrm{Y}$ is not necessarily the same as the distance from the model $\mathrm{Y}$ to $\mathrm{X}$. Our algorithm thus computes four distance values, i.e., two elastic distance values for each of the two orientation configurations, and chooses the smallest of them for the dissmilarity value.

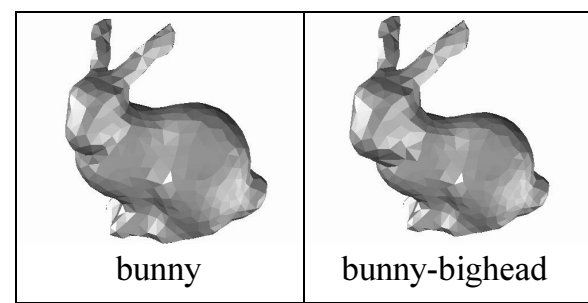

Figure 7. The bunny and the bunny-bighead models.

\section{Experiments and results}

We implemented the proof-of-concept system using $\mathrm{C}++$ on a Linux operating system. For the experiment, we used 262 VRML models we have collected from the Internet. For the experiments presented below, we generated 20,000 points on each model to normalize pose of the model and calculate the feature vector $\mathbf{F}$.

\subsection{Shape features and distance measures}

The first set of experiments compared distance measures using 6 models shown in Figure 8. Figure 8a to Figure 8d are variations of bunny model. The models in Figure $8 \mathrm{~b}$ and Figure $8 \mathrm{c}$ are generated from (a) by locally enlarging a part of the model of Figure 8a. The model in Figure 8d has a shape basically similar to the one in Figure $8 \mathrm{a}$, but has much higher polygon count and a part of the model is cut off. Similarly, the model of Figure $8 \mathrm{f}$ is created by locally scaling the original model of Figure 8e.

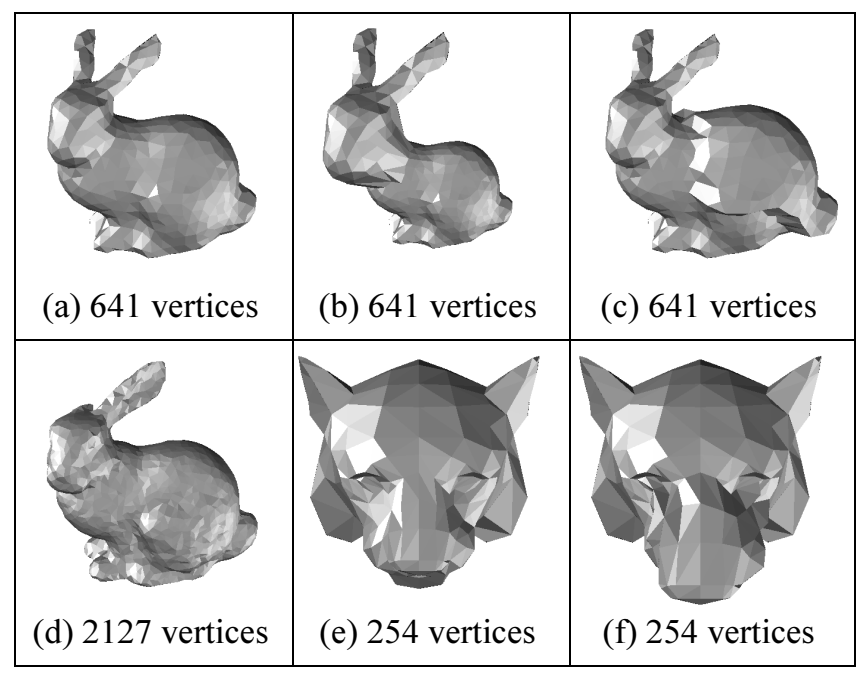

Figure 8. Models used to evaluate distance measures. The results are shown in Table 1, Table 2, and Table 3.

Table 1 shows the dissimilarity values computed using Euclidean distance while Table 2 shows the dissimilarity values computed using the elastic-matching distance. In both Table 1 and Table 2, the number of analysis windows 
is 15 . Bold letters in both of the tables indicate lower dissimilarity values. Note that a direct comparison of values between Table 1 and Table 2 is meaningless for the dissimilarity computation methods are different.

In each of Table 1 and Table 2, the dissimilarity values mostly conform to our expectation. Dissimilarity values are smaller if the comparison is within the same group, i.e., either the bunny group or the tiger group.) Dissimilarity values are higher if the comparison is between the groups, i.e., comparing one of the bunnies with one of the tigers.

Table 3 shows the dissimilarity values for the same set of models using 127 analysis windows, causing each one of the analysis windows to become narrow. In this case, dissimilarity values show some anomalies; the tiger group and the bunny group are not clearly separated. This is due to the fact that a narrow analysis window has relatively few points, around 150 on average, introducing noise into the shape feature vectors.

Table 1. Euclidean distance, number of analysis windows 15.

\begin{tabular}{|c|c|c|c|c|c|c|}
\hline & (a) & (b) & (c) & (d) & (e) & (f) \\
\hline (a) & 0 & 0.199 & 0.101 & 0.146 & 0.263 & 0.292 \\
\hline (b) & 0.199 & 0 & 0.223 & 0.213 & 0.303 & 0.299 \\
\hline (c) & 0.101 & 0.223 & 0 & 0.151 & 0.261 & 0.275 \\
\hline (d) & 0.146 & 0.213 & 0.151 & 0 & 0.266 & 0.279 \\
\hline (e) & 0.263 & 0.303 & 0.261 & 0.266 & $\mathbf{0}$ & 0.138 \\
\hline (f) & 0.292 & 0.299 & 0.275 & 0.279 & 0.138 & 0 \\
\hline
\end{tabular}

Table 2. Elastic-matching distance, number of analysis windows 15 .

\begin{tabular}{|c|c|c|c|c|c|c|}
\hline & (a) & (b) & (c) & (d) & (e) & (f) \\
\hline (a) & 0 & 0.152 & 0.071 & 0.124 & 0.165 & 0.190 \\
\hline (b) & 0.152 & 0 & 0.162 & 0.157 & 0.216 & 0.211 \\
\hline (c) & 0.071 & 0.162 & 0 & 0.114 & 0.179 & 0.195 \\
\hline (d) & 0.124 & 0.157 & 0.114 & 0 & 0.186 & 0.196 \\
\hline (e) & 0.165 & 0.216 & 0.179 & 0.186 & 0 & 0.084 \\
\hline (f) & 0.190 & 0.211 & 0.195 & 0.196 & 0.084 & $\mathbf{0}$ \\
\hline
\end{tabular}

Table 3. Elastic matching distance, number of analysis windows 127.

\begin{tabular}{|c|c|c|c|c|c|c|}
\hline & (a) & (b) & (c) & (d) & (e) & (f) \\
\hline (a) & $\mathbf{0}$ & 0.041 & 0.030 & 0.036 & 0.047 & 0.040 \\
\hline (b) & 0.041 & 0 & 0.042 & 0.048 & 0.043 & 0.043 \\
\hline (c) & 0.030 & 0.042 & 0 & 0.038 & 0.038 & 0.046 \\
\hline (d) & 0.036 & 0.048 & 0.038 & 0 & 0.031 & 0.039 \\
\hline (e) & 0.047 & 0.043 & 0.038 & 0.031 & 0 & 0.032 \\
\hline (f) & 0.040 & 0.043 & 0.046 & 0.039 & 0.032 & 0 \\
\hline
\end{tabular}

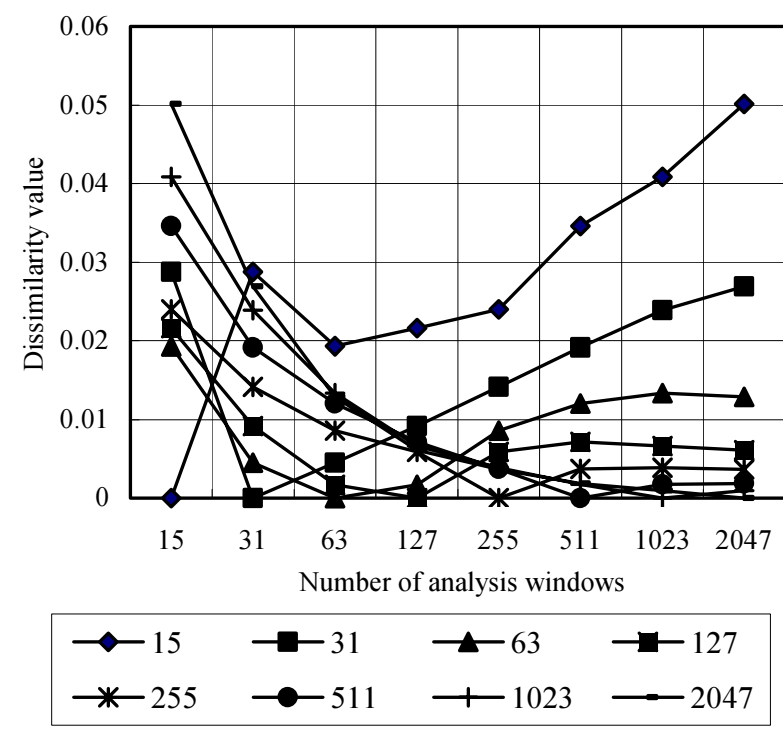

Figure 9. Distance to self computed using various numbers of analysis windows.

Figure 9 shows the plot of dissimilarity value of a model to itself computed using 8 different numbers of analysis windows. For the computation, elastic matching dissimilarity value is used. (Euclid distance is not defined for a pair of vectors having different dimensions.) Ideally, all the dissimilarity values should be zero. However, due to the noise discussed above, positive non-zero dissimilarity values occur. Of the combinations, 64 analysis windows appear to have the lowest dissimilarity value to self. Note that if the number of analysis windows is too small, sensitivity of the measure to the shape difference will decrease. As a compromise, we chose to use 64 analysis windows for the experiments that followed.

\subsection{Shape similarity-based search}

In this experiment, we tried to retrieve models close in shape to each of the 4 models shown in Figure 10. For each model presented as an example, the system retrieved 8 models having the lowest dissimilarity values in the database. We compared two dissimilarity measure based on Euclidean and elastic matching distances. In this experiments, all the three statistics have the equal weight. As mentioned, the search was performed on the database containing 261 (loosely defined) mesh models.

The results of the experiments are shown in Figure 11 through to Figure 14. In all of the cases, models having shapes more or less close to the query model were retrieved. The shape feature parameterized along principal axes appears to work well for the shapes having some form of rotational symmetry. But for other models, such as a curly 
Chinese-dragon model, or a human figure in a contorted body configuration, the shape feature proposed in this paper did not perform well.

The Euclidean and elastic-matching dissimilarity measures produced results that are similar but not identical. Query results of Figure 12a and Figure 12b illustrate well the difference between the two dissimilarity measures. Given a chess piece, the search using the elastic-matching distance retrieved models having wide ends and a narrow waist, with little regard to the overall "proportion". For example, the chess piece (query) and the light stand with a long thin middle part came out close. As expected, the elastic matching effectively skipped the middle part by its "elasticity", trying to match the flaring shape features at ends. The Euclidean distance, on the other hand, retrieved shapes whose overall proportions are closer to the original chess piece, e.g., bolts and screws.

\subsection{Computation time}

Computation time required to search 8 closest models out of a database containing 261 models using our method is tabulated in Table 4. Most of the time is spent on computing the feature vector of the query model, while very little time is spent on linearly matching all the candidates. (As mentioned before, no indexing structure or hierarchical search scheme is implemented.)

\section{Summary and conclusion}

In this paper, we proposed and evaluated several key components for a shape similarity search and retrieval system. The system expects, as inputs, VRML-like 3D mesh models. Our method is tolerant so it accepts models having geometrical degeneracies or multiple disconnected components, for example. As shape features, the method employs three statistics that are discretely parameterized along the principal axes of inertia of the model. The method first normalizes the poses of the models by using the principal axes of inertia of the model. The three statistics are the moment of inertia, the average distance from the surfaces to the axis, and the variance of the distance from the surface to the axis. Both the pose normalization and shape feature computation assumes that the polygonal surfaces to have uniform density, and approximates it by using a quasi-Monte Carlo approach. For dissimilarity computation, we experimented with two methods, the simple Euclidean distance and an elastic matching algorithm that is implemented using dynamic programming. A set of experiments showed that our shape feature and dissimilarity computation methods are promising candidates for inclusion in our future shape similarity search system.

Obviously, there are many things to be done in the field

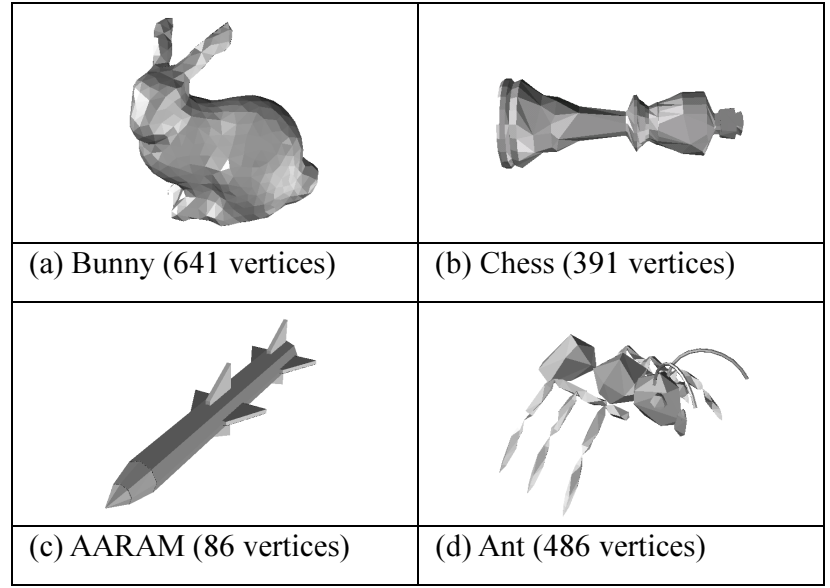

Figure 10. Models queried in the experiments.

Table 4. Time to compute and rank dissimilarity values for all the 261 models in a database for the queried model.

\begin{tabular}{|r||r|r|}
\hline \multirow{2}{*}{$\begin{array}{c}\text { Number of } \\
\text { analysis } \\
\text { windows }\end{array}$} & \multicolumn{2}{|c|}{ Search and retrieval time } \\
\cline { 2 - 3 } & Euclidean distance & Elastic matching \\
\hline \hline 15 & $0 \mathrm{~s}$ & $0 \mathrm{~s}$ \\
\hline 31 & $0 \mathrm{~s}$ & $1 \mathrm{~s}$ \\
\hline 63 & $1 \mathrm{~s}$ & $2 \mathrm{~s}$ \\
\hline 127 & $3 \mathrm{~s}$ & $3 \mathrm{~s}$ \\
\hline 255 & $6 \mathrm{~s}$ & $8 \mathrm{~s}$ \\
\hline 511 & $13 \mathrm{~s}$ & $16 \mathrm{~s}$ \\
\hline 1023 & $25 \mathrm{~s}$ & $32 \mathrm{~s}$ \\
\hline 2047 & $51 \mathrm{~s}$ & $64 \mathrm{~s}$ \\
\hline
\end{tabular}

of shape similarity search.

The foremost on the list of issues is the further development of shape features. As the demand for the shape similarity search is diverse, the authors think that not one but a set of multiple shape features must be recruited for an effective shape similarity search. We are interested in the shape features that won't require pose-normalization. We are also interested in shape features that could capture topology of the shape and are applicable to not-so-welldefined shape definitions.

The system will also require some mechanisms, e.g., a learning-based mechanism a la Elad et al. [Elad01], to accommodate a user's preference and taste in retrieving shapes the user wants. We intend to work on this aspect of the problem.

As the last note, in this field of research must find a set of standard data and performance evaluation method so that we can objectively compare the shape similarity search algorithms we develop. This is much more difficult to accomplish than a similar task in the field of image content based search and retrieval, due in part to the diverse 3D shape representations. 


\section{Acknowledgements}

This research has been funded in part by the grant No. 12680432 from the Ministry of Education, Culture, Sports, Sciences, and Technology of Japan, and by the grant from the Okawa Foundation for Information and Telecommunications. We thank Prof. Shigeo Takahashi for discussion and providing us with the software collection gmtools on which our prototype system is based.

\section{References}

[Burges98] C. Burges, A Tutorial on Support Vector Machines for Pattern Recognision, Data Mining and Knowledge Discovery, 2, pp. 1-47, 1998.

[Corney02] J. Corney, H. Rea, D. Clark, John Pritchard, M. Breaks, R. MacLeod, Coarse Filter for Shape Matching, IEEE $C G \& A$, pp. 65-73, May/June, 2002.

[Elad00] M. Elad, A. Tal, S. Ar. Directed Search in A 3D Objects Database Using SVM, HP Laboratories Israel Technical Report, HPL-2000-20 (R.1), August, 2000.

[Gottschalk96] Gottschalk, S., Lin, M.C., Manocha, D., OBBTree: A Hierarchical Structure for Rapid Interference Detection, Proc. SIGGRAPH '96, pp. 171-180, 1996.

[Hilaga01] M. Hilaga, Y. Shinagawa, T. Kohmura, and T. Kunii. Topology Matching for Fully Automatic Similarity Estimation of 3D Shapes. Proc. SIGGRAPH 2001, pp. 203-212, Los Angeles, USA. 2001.

[Ibato02] Masatoshi Ibato, Tomo Otagiri, Ryutarou Ohbuchi, Shape-similarity Search of Three-Dimensional Models Based on Subjective Measures, IPSJ SIG report on Graphics\&CAD, Vol. 2002, No. 16 (2002-CG-106), pp. 25-30, February, 2002.

[Igarashi99] Takeo Igarashi, Hidehiko Tanaka, Satoshi Matusoka, Teddy: A Sketching Interface for 3D Freeform Design, Proc. SIGGRAPH' '99, pp. 409-416, 1999.

[Keim99] D. Keim, Efficient Geometry-based Similarity Search of 3D Spatial Databases, Proc. ACM SIGMOD Int. Conf. On Management of Data, pp. 419-430, Philadelphia, PA., 1999.

[McWherter01] D. McWherter, M. Peabody, W. Regli, A. Shokoufandeh, Transformation Invariant Shape Similarity Comparison of Solid Models, Proc. ASME DETC '2001, September 2002, Pittsburgh, Pennsylvania.
[Mukai02] S. Mukai, S. Furukawa, M. Kuroda, An Algorithm for Deciding Similarities of 3-D Objects, Proc. ACM Symposium on Solid Modeling and Applications 2002, Saarbrücken, Germany, June 2002.

[Novotni01] M. Novotni, R. Klein. A Geometric Approach to 3D Object Comparison. Proc. Int'l Conf. on Shape Modeling and Applications 2001, pp. 167-175, Genova, Italy, May, 2001.

[Osada01] Osada, T. Funkhouser, B. Chazelle, D. Dobkin. Matching 3D Models with Shape Distributions. Proc. Int'l Conf. on Shape Modeling and Applications 2001, pp. 154-166, Genova, Italy, May, 2001.

[Paquet97] E. Paquet and M. Rioux, Nefertiti: a Query by Content Software for Three-Dimensional Databases Management, Proc. Int'l Conf. on Recent Advances in 3-D Digital Imaging and Modeling, pp. 345-352, Ottawa, Canada, May 12-15, 1997.

[Paquet00] E. Paquet, A. Murching, T. Naveen, A. Tabatabai, M. Roux. Description of shape information for 2-D and 3-D objects. Signal Processing: Image Communication, 16:103-122, 2000.

[Regli00] W. Regli, V. Cicirello, Managing Digital Libraries for Computer-Aided Design, Computer Aided Design, pp. 110-132, Vol. 32, No. 2, 2000.

[Suzuki98] M. T. Suzuki, T. Kato, H. Tsukune. 3D Object Retrieval based on subject measures, Proc. $9^{\text {th }}$ Int'l Conf. and Workshop on Database and Expert Systems Applications (DEXA98), pp. 850-856, IEEE-PR08353, Vienna, Austria, Aug. 1998.

[Suzuki00] M. T. Suzuki, T. Kato, N. Otsu. A similarity retrieval of 3D polygonal models using rotation invariant shape descriptors. IEEE Int. Conf. on Systems, Man, and Cybernetics (SMC2000), Nashville, Tennessee, pp. 2946-2952, 2000.

[Vapnik98] V. N. Vapnik. Statistical Learning Theory. Wiley, 1998.

[Vapnik99] V. N. Vapnik. The Nature of Statistical Learning Theory, Second Edition. Springer, 1999.

[Veltkamp01] R. C. Veltkamp. Shape Matching: Similarity Measures and Algorithms, invited talk, Proc. Int'l Conf. on Shape Modeling and Applications 2001, pp. 188-197, Genova, Italy, May, 2001.

[Vranić01] D. V. Vranić, D. Saupe, and J. Richter. Tools for 3Dobject retrieval: Karhunen-Loeve Transform and spherical harmonics. Proc. of the IEEE 2001 Workshop on Multimedia Signal Processing, Cannes, France, pp. 293-298, October 2001. 


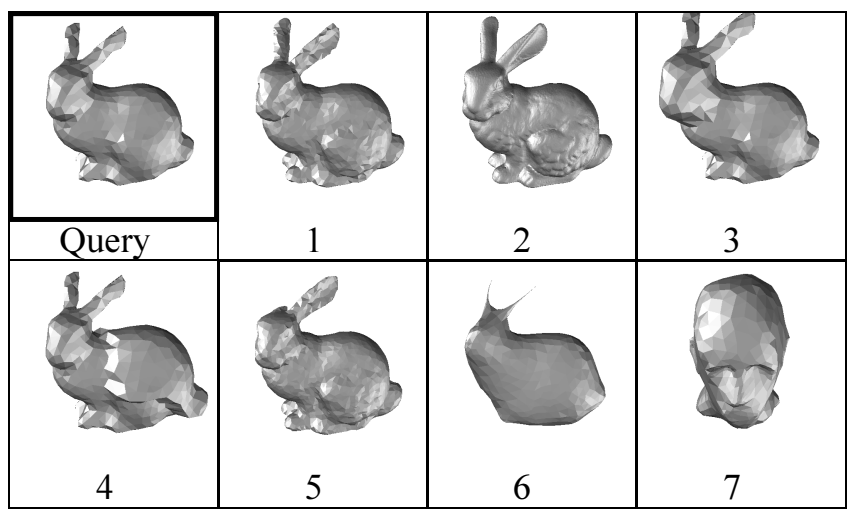

Figure 11a. Euclidean distance, 63 analysis windows.

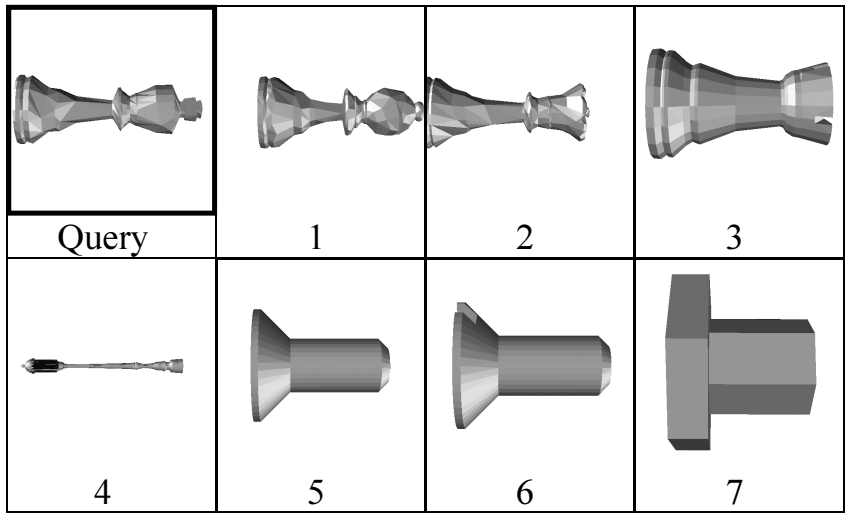

Figure 12a. Euclidean distance, 63 analysis windows.

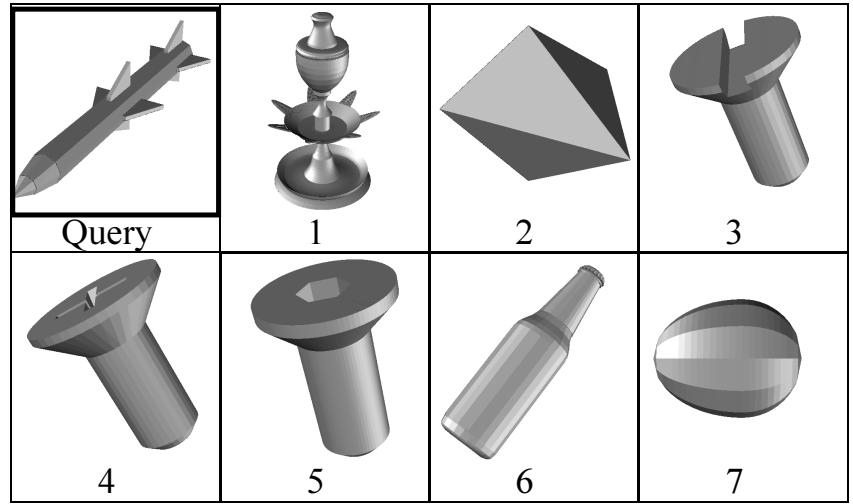

Figure 13a. Euclidean distance, 63 analysis windows.

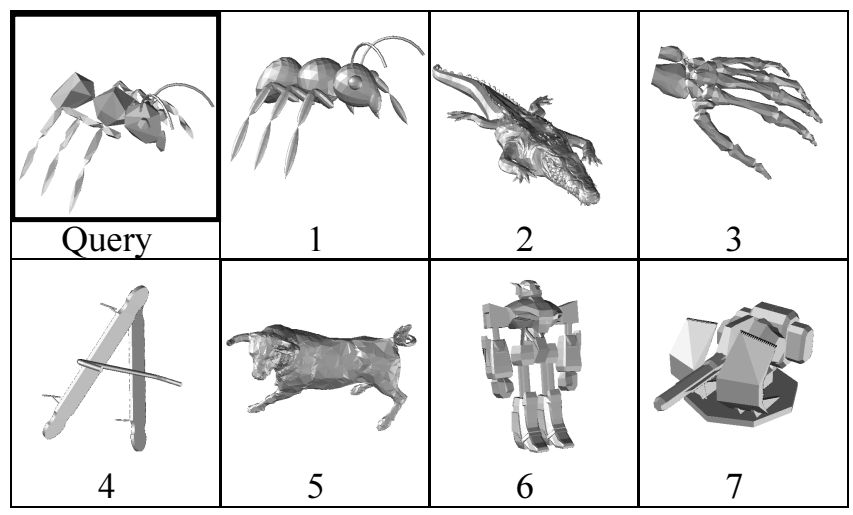

Figure 14a. Euclidean distance, 63 analysis windows.

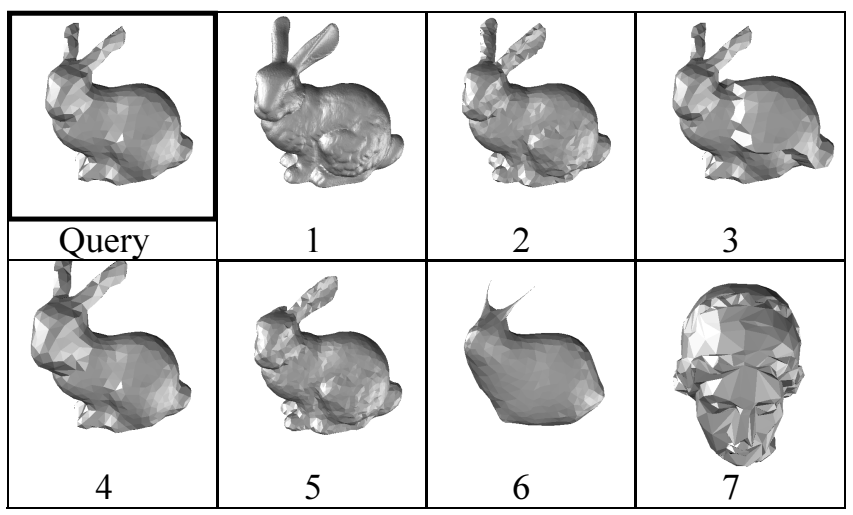

Figure 11b. Elastic matching, 63 analysis windows.

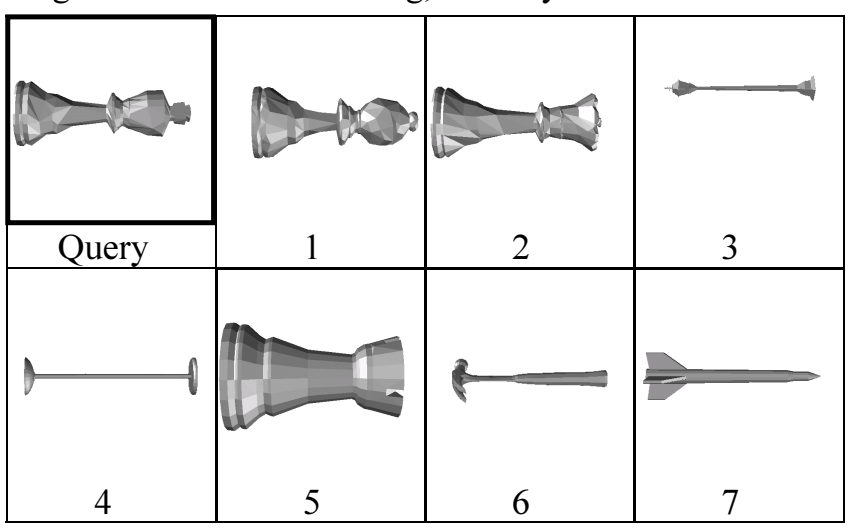

Figure 12b. Elastic matching, 63 analysis windows.

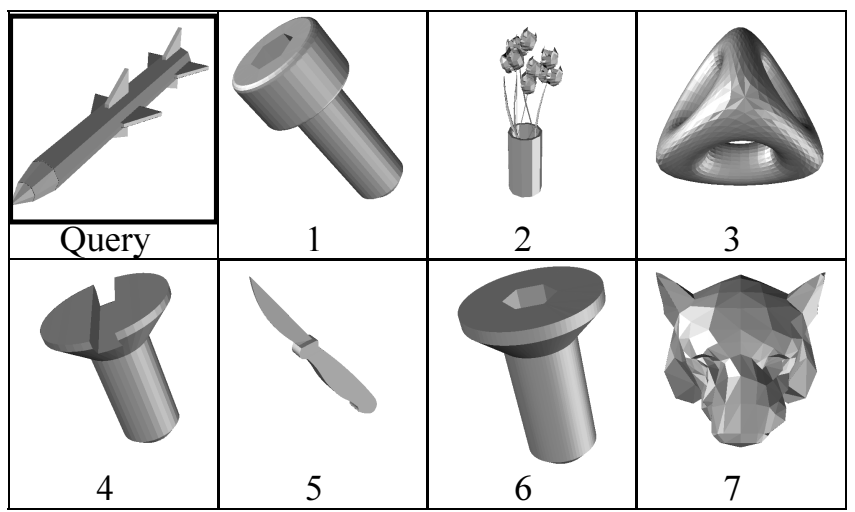

Figure 13b. Elastic matching, 63 analysis windows.

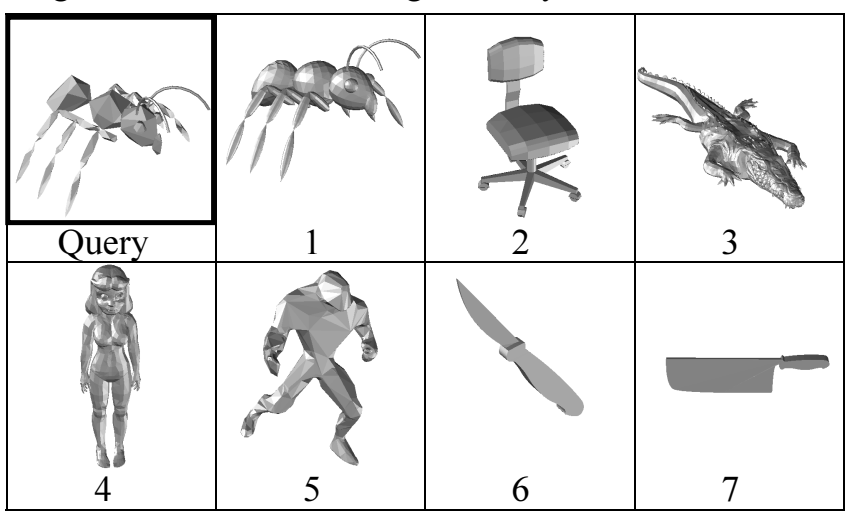

Figure 14b. Elastic matching, 63 analysis windows. 